\title{
Quantum Mechanical Search and Harmonic Perturbation
}

\author{
Jie-Hong R. Jiang, ${ }^{1,4}$ Dah-Wei Chiou, ${ }^{2}$ and Cheng-En $\mathrm{Wu}^{3}$
}

Received: February 5, 2007; accepted: August 5, 2007; Published online: September 21, 2007

Perturbation theory in quantum mechanics studies how quantum systems interact with their environmental perturbations. Harmonic perturbation is a rare special case of time-dependent perturbations in which exact analysis exists. Some important technology advances, such as masers, lasers, nuclear magnetic resonance, etc., originated from it. Here we add quantum computation to this list with a theoretical demonstration. Based on harmonic perturbation, a quantum mechanical algorithm is devised to search the ground state of a given Hamiltonian. The intrinsic complexity of the algorithm is continuous and parametric in both time $T$ and energy E. More precisely, the probability of locating a search target of a Hamiltonian in $N$-dimensional vector space is shown to be $1 /\left(1+c N E^{-2} T^{-2}\right)$ for some constant c. This result is optimal. As harmonic perturbation provides a different computation mechanism, the algorithm may suggest new directions in realizing quantum computers.

KEY WORDS: quantum computation; complexity; Grover database search; harmonic perturbation.

PACS: 03.67.Lx.

\section{INTRODUCTION}

Quantum physics can in principle speed up solving the unsorted-database search problem with a quadratic improvement over classical algorithms, as was first demonstrated by $\operatorname{Grover}^{(1)}$. This problem was originally formulated as to identify a target item in the fewest queries to a black-box database. An important reformulation by Farhi et al. ${ }^{(2,3)}$ phrased the problem as to search the target state with some special eigenvalue of a given

\footnotetext{
${ }^{1}$ Department of Electrical Engineering, National Taiwan University, Taipei 10617, Taiwan.

${ }^{2}$ Department of Physics, University of California, Berkeley, CA 94720, USA.

${ }^{3}$ Department of Physics, National Tsing Hua University, Hsinchu 30013, Taiwan.

${ }^{4}$ To whom correspondence should be addressed. E-mail: jhjiang@cc.ee.ntu.edu.tw
} 
Hamiltonian $\mathcal{H}$, which represents the database. In particular, constant and adiabatic perturbations were proposed in ${ }^{(2)}$ and ${ }^{(3)}$, respectively, for quantum search. These methods yield the same quadratic speed-up as Grover's construct. ${ }^{(4,5)}$ Moreover, the adiabatic computation is equivalent to standard quantum computation (in terms of unitary transformations). ${ }^{(6)}$ Although some physical implementations have been demonstrated in realizing quantum search algorithms, e.g. ${ }^{(7-9)}$, they may not be scalable to solve large problem instances without some fundamental breakthroughs. ${ }^{(10)}$ Searching alternative computation models may suggest new ways of building quantum computers.

Despite the success of the constant and adiabatic perturbations in quantum search, the applicability of perturbations based on fast timevarying Hamiltonians remains an open problem. This paper exploits harmonic perturbation for quantum computation. A new computation model is proposed, inspired by the well-studied harmonic perturbation of twostate systems in quantum mechanics. By preparing a system in one or the other of its two states initially, such perturbation induces an absorption-emission cycle (the phenomenon of periodic oscillation of the probability for the system being found at one of its two states) at the resonance condition. More specifically, consider a two-state physical system of Hamiltonian $\mathcal{H}=E_{1}|1\rangle\left\langle 1\left|+E_{2}\right| 2\right\rangle\langle 2|$, with $E_{2}>E_{1}$, in a sinusoidal potential $\mathcal{V}(t)=\gamma e^{i \omega t}|1\rangle\left\langle 2\left|+\gamma e^{-i \omega t}\right| 2\right\rangle\langle 1|$. The state evolution of the system is governed by the Schrödinger equation

$$
i \frac{\partial \psi(t)}{\partial t}=\widetilde{\mathcal{H}}(t) \psi(t)
$$

with $\psi(t)=c_{1}(t)|1\rangle+c_{2}(t)|2\rangle$ and $\widetilde{\mathcal{H}}(t)=\mathcal{H}+\mathcal{V}(t)$. For the initial condition $c_{1}(0)=0$ and $c_{2}(0)=1$, the respective probabilities of finding the system in states $|1\rangle$ and $|2\rangle$ are of exact solutions (Rabi's formula; see, e.g., ${ }^{(11)}$ )

$$
\begin{aligned}
\left|c_{1}(t)\right|^{2} & =\frac{\gamma^{2}}{\gamma^{2}+\left(\omega-\omega_{21}\right)^{2} / 4} \sin ^{2}(\Omega t) \\
\left|c_{2}(t)\right|^{2} & =1-\left|c_{1}(t)\right|^{2}
\end{aligned}
$$

where $\omega_{21} \equiv\left(E_{2}-E_{1}\right)$ and $\Omega \equiv \sqrt{\gamma^{2}+\frac{\left(\omega-\omega_{21}\right)^{2}}{4}}$. At resonance, $\omega=\omega_{21}$, the probability of finding the system in the ground state $|1\rangle$ oscillates with period $\pi / \gamma$, and reaches 1 at time $(2 k+1) \pi /(2 \gamma), k=0,1,2, \ldots$ This phenomenon reveals the potential usefulness of harmonic perturbation in searching the ground state of a given Hamiltonian even with arbitrarily multiple states. 
Intuitively harmonic perturbation to a general multiple-state system may be used as a mechanism searching the ground state of the corresponding Hamiltonian. Knowing the energy gap between the initial state and ground state, one can apply a perturbation at the resonance frequency to induce a probability oscillation such that the system swings between these two states. If a measurement is performed at the right time, the system situates definitely in its ground state. Based on this intuition, we devise a quantum search algorithm. An analysis shows that, given a Hamiltonian in $N$-dimensional vector space with energy gap $E$ between its ground state and the other $N-1$ excited states, our algorithm finds the ground state in time $T$ with probability $\operatorname{Pr}=1 /\left(1+c N E^{-2} T^{-2}\right)$ for some constant $c$. Therefore, to search the right target with a constant high probability independent of $N$, the time-energy product complexity matches prior known $\Theta(\sqrt{N})$ result ${ }^{(12)}$ of other quantum search algorithms. $^{(1-3)}$ Note that, due to the time-energy duality, it is not meaningful to speak about only time or energy complexity, regardless of the other.

\section{QUANTUM SEARCH ALGORITHMS USING HARMONIC PERTURBATION}

We formulate the database search problem as follows. Given a Hamiltonian $\mathcal{H}=\Sigma_{j=1}^{N} E_{j}|j\rangle\langle j|$, we are asked to find state $|g\rangle$ such that $E_{g}$ is the minimum among $E_{j}$ 's, i.e., $|g\rangle$ is the ground state. In the sequel, we assume any state $|j\rangle$ is a configuration of $n$ binary digits; thus, $N=2^{n}$. Moreover, we assume that the state of the underlying physical system is measurable such that its $n$-bit configuration (e.g., spin orientations of spin- $1 / 2$ particles measured along some axis) is completely determined, and that its corresponding energy under $\mathcal{H}$ can be observed thereafter. Unless otherwise stated, we shall focus on the energy distribution of Grover's search problem, and assume one out of the $N$ states is the ground state of energy 0 and the other $N-1$ states are excited states of energy $E>0$. In our algorithm, we consider $E$ is adjustable in analyzing energy complexity.

Below we show the new application of harmonic perturbation in quantum database search. In principle, if the perturbation potential is designed properly, knowing the initial (excited) state and energy gap of a given $N$-state system, one can apply the resonance frequency to induce an oscillatory transition almost solely between the initial state and the ground state, similar to the two-state case. Measuring the system at the 


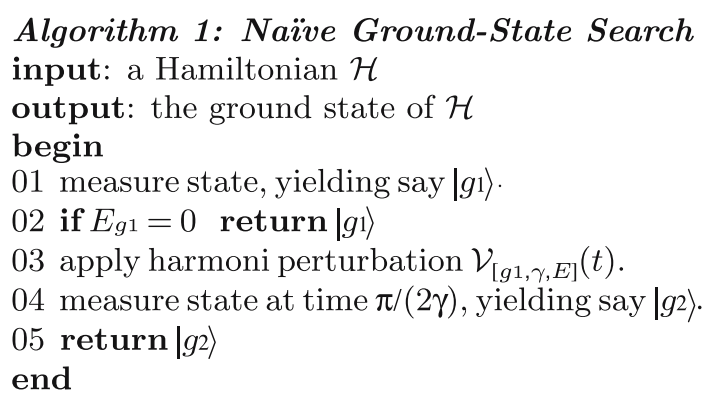

Fig. 1. The procedure of a naïve quantum search.

right time achieves the highest probability of locating the ground state. How this peak probability is related to $N$ is our main concern. To gain an insight on the optimality limit of quantum search using harmonic perturbation, we begin with a simple trial (in Fig. 1) and then proceed with an optimized procedure (in Fig. 4).

\subsection{A Trial Algorithm}

Figure 1 sketches a (non-optimal) quantum search procedure. The algorithm starts with a measurement in the state basis to enforce the underlying physical system collapsing to some state, say $\left|g_{1}\right\rangle$. If the corresponding eigenenergy of the input Hamiltonian $\mathcal{H}$ in state $\left|g_{1}\right\rangle$ equals 0 , the algorithm has found the target and returns $\left|g_{1}\right\rangle$ immediately at Step 2. Otherwise, harmonic perturbation is applied using the sinusoidal potential $\mathcal{V}_{[j, \gamma, \omega]}(t)$ with

$$
\left\langle p\left|\mathcal{V}_{[j, \gamma, \omega]}(t)\right| q\right\rangle= \begin{cases}\gamma e^{i \omega t} & \text { if } q=j \text { and } q \neq p \\ \gamma e^{-i \omega t} & \text { if } p=j \text { and } p \neq q \\ 0 & \text { otherwise }\end{cases}
$$

for indices $p, q=1, \ldots, N$. By replacing index $j$ with $g_{1}$ and letting $\omega=E$, the perturbation $\mathcal{V}_{\left[g_{1}, \gamma, E\right]}(t)$ at Step 3 induces an oscillatory probability for the system swinging mainly between state $\left|g_{1}\right\rangle$ and the unknown ground state. The algorithm measures, at Step 4, the state of the system at time $\pi /(2 \gamma)$, when the system situates in the ground state with the highest probability.

We analyze the condition under which this peak probability is independent of the effect of $N$ and is close to 1 . For $N=2$, the returned state $\left|g_{2}\right\rangle$ of the algorithm is the ground state with certain. However, it is not the case for $N>2$. To see why, we solve the Schrödinger equation of 
the $N$-state system with Hamiltonian $\widetilde{\mathcal{H}}(t)=\mathcal{H}+\mathcal{V}_{\left[g_{1}, \gamma, E\right]}(t)$. To simplify the discussion, assume without loss of generality that $|1\rangle$ is the ground state and $\left|g_{1}\right\rangle=|2\rangle$ is the initial state. Let $c_{k}(t)$ denote the probability amplitude of state $|k\rangle$ at time $t$. Then the original $N$ first-order differential equations from equation (1) can be reduced to three due to the equivalence of $c_{3}(t), c_{4}(t), \ldots, c_{N}(t)$. Hence, at resonance $\omega=E \equiv \omega_{R}$, the reduced equations in terms of $b_{k}(t) \equiv e^{i E_{k} t} c_{k}(t)$ are

$$
\begin{aligned}
i \dot{b_{1}}(t) & =\gamma b_{2}(t) \\
i \dot{b_{2}}(t) & =\gamma b_{1}(t)+(N-2) \gamma e^{-i \omega_{R} t} b_{3}(t) \\
i \dot{b_{3}}(t) & =\gamma e^{i \omega_{R} t} b_{2}(t)
\end{aligned}
$$

To solve $\left|b_{1}(t)\right|^{2}$ and thus $\left|c_{1}(t)\right|^{2}$, apply Laplace transform $\mathcal{L}$ on these equations and solve for $B_{1}(s) \equiv \mathcal{L}\left\{b_{1}(t)\right\}$ with initial conditions $b_{2}(0)=1$ and $b_{k}(0)=0$ for $k \neq 2$. We derive

$$
B_{1}(s)=\frac{-i \gamma}{\left(s^{2}+\gamma^{2}\right)} \frac{1}{\left(1+\frac{\gamma^{2}}{\left(s^{2}+\gamma^{2}\right)} \Lambda_{1}\right)}
$$

where $\Lambda_{1} \equiv(N-2) s /\left(s+i \omega_{R}\right)$. From the inverse Laplace transform of $B_{1}(s)$, the exact solution of $c_{1}(t)$ can be derived. For $N=2$ and thus $\Lambda_{1}=0, c_{1}(t)$ reduces to equation (2). For general $N>2$, we omit listing the sophisticated expression of $c_{1}(t)$ as the trial procedure is not our final destination. Nevertheless, the numerical simulations as shown in Figs. 2 and 3 reveal the performance of Algorithm 1 with respect to $N$. An analysis suggests that, for a fixed constant $\gamma$ (among other possibilities), to maintain a constant peak probability, $\operatorname{Pr}=\max _{t}\left|c_{1}(t)\right|^{2}$, we need $\omega_{R} \propto N$. Thus, the algorithm has time complexity $O(1)$ due to the fixed perturbation amplitude $\gamma$, and has energy complexity $O\left(\|H\|_{2}+\|\mathcal{V}\|_{2}\right)=O\left(\omega_{R}+\right.$ $\gamma \sqrt{N})=O(N)$ for $\omega_{R} \in O(N)$. This constant complexity in time and linear complexity in energy can be explained in the $s$-domain by observing that $N$ and $\omega_{R}$ in $\Lambda_{1}$ of equation (7) are of a first-order relation. That is, by maintaining $N / \omega_{R}=\epsilon$ for some small constant $\epsilon$, the effect of $\Lambda_{1}$ is negligible and $\left|c_{1}(t)\right|^{2} \approx \sin ^{2}(\gamma t)$. Hence, the algorithm achieves the same linear resource complexity as the classical algorithm for database search.

As a digression, we noticed that the recent work ${ }^{(13)}$ presented an algorithm on quantum search with resonance, where the perturbation is very similar to $\mathcal{V}_{[j, \gamma, \omega]}(t)$ of Algorithm 1 and thus the algorithm is unlikely to be superior to the classical counterpart. The misconceived quantum improvement may be due to the ignorance of the time-energy product 


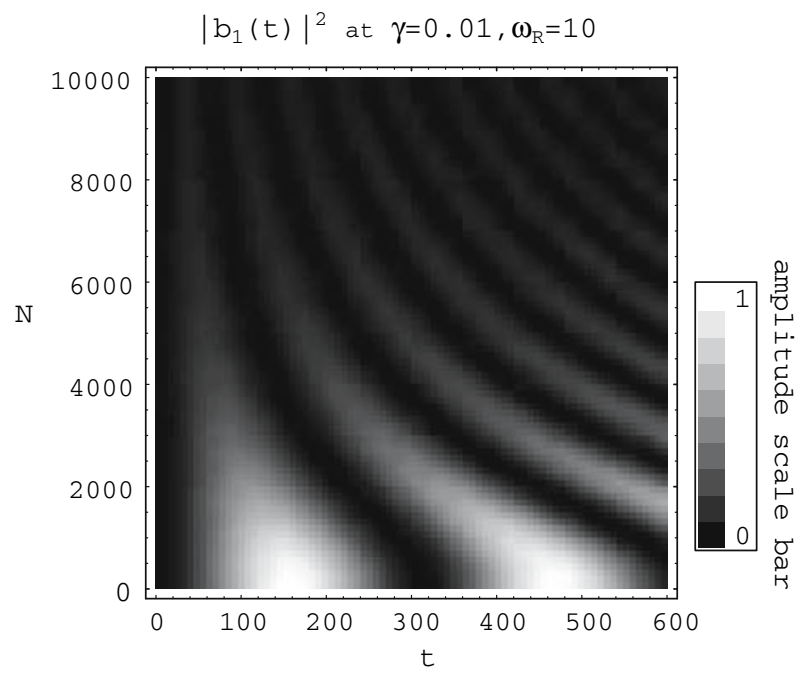

Fig. 2. For $\gamma=0.01, \omega_{R}=10$, the figure plots $\left|b_{1}(t)\right|^{2}$ of Algorithm 1 under various $N$ and $t$. It shows that $\max _{t}\left|b_{1}(t)\right|^{2}$ decreases and $\left|b_{1}(t)\right|^{2}$ oscillates faster as $N$ gets larger.

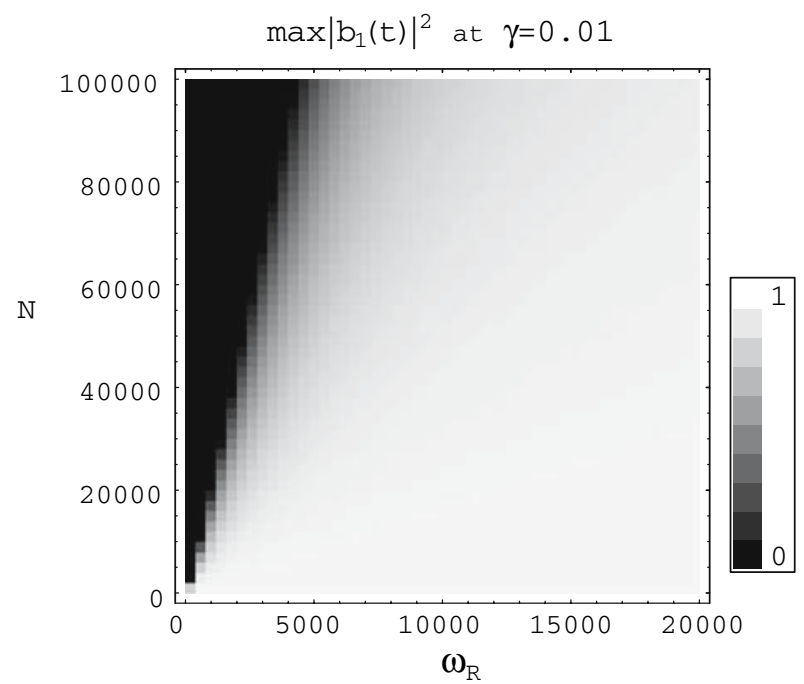

Fig. 3. For $\gamma=0.01$, the figure plots $\max _{t}\left|b_{1}(t)\right|^{2}$ of Algorithm 1 under various $N$ and $\omega_{R}$. It shows that letting $\omega_{R} \propto N$ maintains $\max _{t}\left|b_{1}(t)\right|^{2}$ at some constant value. 


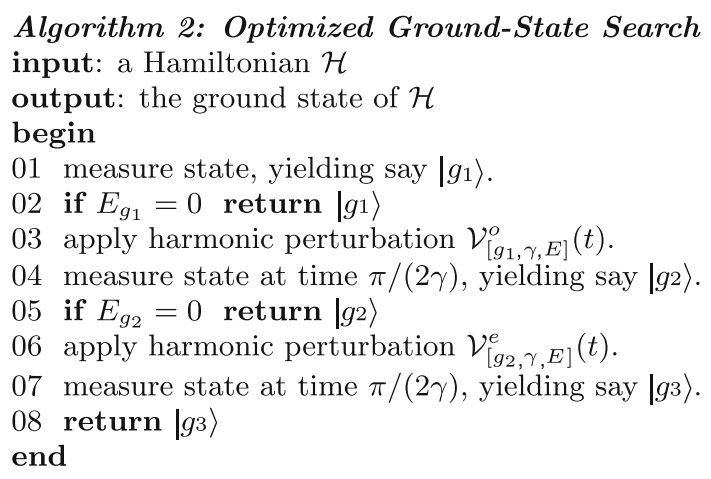

Fig. 4. The procedure of an optimized quantum search.

complexity as well as due to over-simplified analysis. ${ }^{5}$ Moreover, the formulation assumed that the eigenenergy of the search target is unique and known a priori. However, under this assumption, the energy eigenstates could be pre-computed; the target eigenstate can be known once the eigenenergy is specified without even resort to quantum search. (In contrast, we assume that the excited energy eigenstates are degenerate and there is another observable commute with the Hamiltonian that further determines the states.)

\subsection{A Refined Algorithm}

Based on the insight from the $s$-domain analysis, we obtain an optimized algorithm. Fig. 4 sketches a refined quantum search algorithm similar to that of Fig. 1. It differs from the procedure of Fig. 1 mainly in an additional iteration, and in the applied perturbations $\mathcal{V}_{[j, \gamma, \omega]}^{o}(t)$ and $\mathcal{V}_{[j, \gamma, \omega]}^{e}(t)$, where

\footnotetext{
${ }^{5}$ The underlying Hamiltonians for numerical simulations in ${ }^{(13)}$ were taken from the quantum harmonic oscillator and the two-dimensional quantum rotor, which have eigenenergies $E_{m}=$ $E_{0}(m+1 / 2)$ and $E_{m}=E_{0} m^{2}$, respectively. Since these two Hamiltonians are non-degenerate, the highest (or average) eigenenergies in consideration must be no less than $\Omega(N)$ and $\Omega\left(N^{2}\right)$, respectively, which corresponds to our notion of energy complexity. On the other hand, the Schrödinger equations to be solved were oversimplified and resulted in a problematic conclusion about the complexity improvements. In particular, since energy gaps $\left(E_{i}-\right.$ $\left.E_{j}\right) \gg 1 / \sqrt{N}$ for any $i \neq j$, the probability amplitude $c_{k}(t)$ of any state other than the initial and target states was assumed to be 0 . However, this assumption can be invalid because the effect of $N-2$ such small amplitudes may not be ignored.
} 


$$
\left\langle p\left|\mathcal{V}_{[j, \gamma, \omega]}^{o}(t)\right| q\right\rangle=\left\{\begin{array}{cc}
\gamma e^{i \omega t} & \text { if }(p=j \neq q \text { and } q \text { even }) \text { or } \\
& (q=j \neq p \text { and } p \text { odd }) \\
\gamma e^{-i \omega t} & \text { if }(q=j \neq p \text { and } p \text { even }) \text { or } \\
& (p=j \neq q \text { and } q \text { odd }) \\
0 & \text { otherwise }
\end{array}\right.
$$

for indices $p, q=1, \ldots, N$ and $\mathcal{V}_{[j, \gamma, \omega]}^{e}(t)=\mathcal{V}_{[j, \gamma,-\omega]}^{o}(t)$ (i.e., $\mathcal{V}_{[j, \gamma, \omega]}^{o}(t)$ and $\mathcal{V}_{[j, \gamma, \omega]}^{e}(t)$ are conjugate to each other). For instance, $\mathcal{V}_{[2, \gamma, \omega]}^{o}(t)$ in a $4 \times 4$ matrix reads

$$
\left(\begin{array}{cccc}
0 & \gamma e^{i \omega t} & 0 & 0 \\
\gamma e^{-i \omega t} & 0 & \gamma e^{-i \omega t} & \gamma e^{i \omega t} \\
0 & \gamma e^{i \omega t} & 0 & 0 \\
0 & \gamma e^{-i \omega t} & 0 & 0
\end{array}\right)
$$

Because $\mathcal{V}_{[j, \gamma, \omega]}^{o}(t)$ and $\mathcal{V}_{[j, \gamma, \omega]}^{e}(t)$ induce noticeable probability oscillation only when the ground state, say $|g\rangle$, situates at an odd (i.e. $g$ odd) and even (i.e. $g$ even) position, respectively, Algorithm 2 requires one more perturbation-and-measurement iteration than Algorithm 1. If the ground state, not returned in Step 2 of Fig. 4, situates at an odd (respectively even) position, it will be returned at Step 5 (respectively Step 8) almost for sure. The algorithm can be repeated to further enhance its correctness probability. (Notice that, in preparing $\mathcal{V}^{o}$ and $\mathcal{V}^{e}$, we need to specify the initial state for parameter $j$. Accordingly the initialization measurements at Steps 1 and 4 determine the parameters $j$ of $\mathcal{V}_{[j, \gamma, \omega]}^{o}(t)$ and $\mathcal{V}_{[j, \gamma, \omega]}^{e}(t)$, respectively. That is why the perturbation $\mathcal{V}^{e}$ of Step 6 depends on the measurement result $\left|g_{2}\right\rangle$ of Step 4. Alternatively one may discharge this dependence by fixing $j$ to some specific state.)

We analyze the condition under which the probability for Algorithm 2 finding the search target is independent of the effect of $N$ and is close to 1 . To simplify our discussion, again we assume without loss of generality that $|1\rangle$ is the ground state of $\mathcal{H}$ and the first measurement in Step 1 of Fig. 4 yields $\left|g_{1}\right\rangle=|2\rangle$. To compute the probability that the ground state $|1\rangle$ is correctly returned in Step 5, we solve the Schrödinger equation of an $N$-state system with Hamiltonian $\widetilde{\mathcal{H}}(t)=\mathcal{H}+\mathcal{V}_{[2, \gamma, E]}^{o}(t)$. Let $c_{k}(t)$ be the probability amplitude of state $|k\rangle$ at time $t$. Then the original $N$ first-order differential equations can be reduced to four due to the equivalence of odd coefficients $c_{3}(t), \ldots, c_{N-1}(t)$ and the equivalence of even coefficients $c_{4}(t), \ldots, c_{N}(t)$. Hence, at resonance $\omega=E \equiv \omega_{R}$, the reduced equations in terms of $b_{k}(t) \equiv e^{i E_{k} t} c_{k}(t)$ are 


$$
\begin{aligned}
i \dot{b_{1}}(t) & =\gamma b_{2}(t) \\
i \dot{b_{2}}(t) & =\gamma b_{1}(t)+\frac{(N-2)}{2} \gamma\left(e^{-i \omega_{R} t} b_{3}(t)+e^{i \omega_{R} t} b_{4}(t)\right) \\
i \dot{b_{3}}(t) & =\gamma e^{i \omega_{R} t} b_{2}(t) \\
i \dot{b_{4}}(t) & =\gamma e^{-i \omega_{R} t} b_{2}(t)
\end{aligned}
$$

To solve $\left|b_{1}(t)\right|^{2}$ and thus $\left|c_{1}(t)\right|^{2}$, we apply Laplace transform $\mathcal{L}$ on these equations and solve for $B_{1}(s) \equiv \mathcal{L}\left\{b_{1}(t)\right\}$ with initial conditions $b_{2}(0)=1$ and $b_{k}(0)=0, k \neq 2$. We derive

$$
B_{1}(s)=\frac{-i \gamma}{\left(s^{2}+\gamma^{2}\right)} \frac{1}{\left(1+\frac{\gamma^{2}}{\left(s^{2}+\gamma^{2}\right)} \Lambda_{2}\right)}
$$

where $\Lambda_{2} \equiv(N-2) s^{2} /\left(s^{2}+\omega_{R}^{2}\right)$. Taking inverse Laplace transform $\mathcal{L}^{-1}\left\{B_{1}(s)\right\}$ and assuming $N \gg 1$, we get

$$
b_{1}(t) \approx \frac{-i \omega_{R}}{\sqrt{N \gamma^{2}+\omega_{R}^{2}}} \sin \left(\frac{\gamma \omega_{R}}{\sqrt{N \gamma^{2}+\omega_{R}^{2}}} t\right)
$$

By equation (13), the peak probability $\operatorname{Pr}$ of finding the search target equals $\max _{t}\left|c_{1}(t)\right|^{2}=1 /\left(1+N \gamma^{2} \omega_{R}{ }^{-2}\right)$; the period of the probability oscillation of $\left|c_{1}(t)\right|^{2}$ is $\tau=\pi \sqrt{1+N \gamma^{2} \omega_{R}^{-2}} / \gamma$. Since on average the search target can be found by running the algorithm $1 / \mathrm{Pr}$ times each of which takes time $\tau$, the total time complexity is of $\tau / \operatorname{Pr} \in O\left(\left(1+N \gamma^{2} \omega_{R}{ }^{-2}\right)^{\frac{3}{2}} / \gamma\right)$. On the other hand, the energy complexity is of $O\left(\|H\|_{2}+\|\mathcal{V}\|_{2}\right)=O\left(\omega_{R}+\right.$ $\gamma \sqrt{N})$. It can be verified that maintaining a constant $\operatorname{Pr}$ achieves the tightest upper bound of resource complexity. As a result, when $\operatorname{Pr}$ is maintained as a constant (as we shall assume in the sequel), $\gamma$ is the only parameter affecting time complexity $T$. Letting $E$ denote the energy complexity, we can write $\operatorname{Pr}=1 /\left(1+c N E^{-2} T^{-2}\right)$ for some constant $c$. In essence, the time-energy product complexity is of $O(\sqrt{N})$.

The foregoing analysis assumes that the ground state is in an odd position. Suppose that the ground state is rather in some even position. Then $\mathcal{V}_{\left[g_{1}, \gamma, E\right]}^{o}(t)$ of Step 3 does not induce a state oscillation (and thus $\left|g_{2}\right\rangle$ is the same as $\left|g_{1}\right\rangle$ with high probability) while $\mathcal{V}_{\left[g_{2}, \gamma, E\right]}^{e}(t)$ of Step 6 does. In this case, a similar analysis holds to derive the probability that the algorithm returns a correct answer at Step 8.

Since Algorithm 2 achieves the $O(\sqrt{N})$ time-energy product complexity, it is optimal according to the analysis of ${ }^{(2)}$ Below we provide yet another proof using $s$-domain analysis. It may provide an intuition why Algorithm 2 outperforms Algorithm 1. Also we hope that the technique of 
$s$-domain analysis may become a powerful tool for the complexity analysis of other quantum algorithms. As least in our cases, $s$-domain analysis is more intuitive than time-domain analysis.

\section{THE OPTIMALITY LIMIT}

Comparing Algorithms 1 and 2, one might think that the performed modification might seem suspiciously irrelevant as it only affects states $|3\rangle, \ldots,|N\rangle$ in our example. However, this is not true because it in fact affects $|2\rangle$ by equation (9) and thus $|1\rangle$ by equation (8). (Note that Algorithm 2 on perturbation $\mathcal{V}^{o}$, and also $\mathcal{V}^{e}$, applies both $e^{i \omega t}$ and $e^{-i \omega t}$ simultaneously, rather than sequentially. It differs from applying $e^{i \omega t}$ on some states for one search and then applying $e^{-i \omega t}$ on other states for another search. Otherwise, Algorithm 2 would be equivalent to performing Algorithm 1 for several times and has no improvements.) Although the effect may seem obscure from the time-domain Schrödinger equations, it is apparent when solving them in the $s$-domain. The two different phases in $\mathcal{V}^{o}$ and $\mathcal{V}^{e}$ of Algorithm 2 may interfere and simplify $B_{1}(s)$.

To understand the improvement of Algorithm 2 over Algorithm 1, compare equations (7) and (12). We see that the undesirable effect of large $N$ in $\Lambda_{2}$ is nullified by $\omega_{R}^{2}$ while that in $\Lambda_{1}$ is nullified by $\omega_{R}$. As a consequence, for constant $\gamma$, Algorithms 1 and 2 are of energy complexities $O(N)$ and $O(\sqrt{N})$, respectively. Ideally, if we can construct a $\Lambda$ such that the effect of $N$ is cancelled out by $\omega_{R}^{d}$ for a larger exponent $d$, then Pr can be maintained as a constant with a lower resource complexity. Unfortunately, we will now show that $d$ is at most 2, that is, no improvement is possible by introducing more different phases and/or amplitudes to the non-zero entries of $\mathcal{V}_{[j, \gamma, \omega]}^{o}(t)$ and $\mathcal{V}_{[j, \gamma, \omega]}^{e}(t)$. (Note that replacing the zero entries of $\mathcal{V}_{[j, \gamma, \omega]}^{o}(t)$ and $\mathcal{V}_{[j, \gamma, \omega]}^{e}(t)$ with non-zero elements introduces undesirable amplitude leakage to states other than the initial and target ground states. Hence we only need to consider modifying the non-zero entries.)

Consider a general perturbation potential (a Hermitian matrix) with non-vanishing entries only in the row and column indexed by some initial state $|j\rangle$ similar to $\mathcal{V}_{[j, \gamma, \omega]}(t)$. These entries can have arbitrary amplitudes and frequencies. (However, one of the frequencies must equal $\omega_{R}$ such that resonance is possible.) Assuming without loss of generality $|1\rangle$ and $|2\rangle$ to be the ground and initial state, respectively, we solve the corresponding 
Schrödinger equations and can write

$$
B_{1}(s)=\frac{-i \gamma}{\left(s^{2}+\gamma^{2}\right)} \frac{1}{\left(1+\frac{\gamma^{2}}{\left(s^{2}+\gamma^{2}\right)} \Lambda\right)}
$$

with $\gamma$ being the amplitudes for entries $|1\rangle\langle 2|$ and $|2\rangle\langle 1|$, and

$$
\Lambda \equiv s\left(\frac{\alpha_{1}}{s+i \omega_{1}}+\frac{\alpha_{2}}{s+i \omega_{2}}+\cdots+\frac{\alpha_{m}}{s+i \omega_{m}}\right)
$$

where index $m$ is polynomial in $n$ (thus $m \ll N=2^{n}$ ), $\alpha_{j}$ 's are positive real numbers, $\omega_{j}$ 's are of the form $a_{j} \omega_{R} x_{j}$ for real constants $a_{j}$ and $x_{j}$, and $\omega_{j} \neq \omega_{k}$ for $j \neq k$. (Note that $\alpha_{j}$ is obtained from the product of some complex number and its complex conjugate, and thus is positive.)

Theorem 1 Let $d$ equal the largest exponent in terms of $\omega_{R}$ in the denominator of equation (14) minus that in the nominator. Then, $d \leq 2$ for all possible assignments to the constant parameters $\alpha_{j}, a_{j}$, and $x_{j}$ of $\Lambda$.

Since $d=2$ is achieved, the quantum search algorithm of Fig. 4 is optimal.

\section{DISCUSSIONS}

We compare various quantum search algorithms, namely, those of ${ }^{(1-3)}$ and ours, in terms of the generalized problem that the search target appears more than once in the database (i.e., there are multiple ground states in our setup). For ${ }^{(1)}$, the number of appearances needs to be known a priori for accurate search (unless more complicated quantum counting $^{(14)}$ is incorporated); for ${ }^{(2)}$, the method may also seem no easy extension. In contrast, adiabatic computation ${ }^{(3)}$ supports multiple appearances of search target. However, it requires careful analysis to decide valid evolution speed; whether there is quantum speedup may depend on the instance to be solved. On the other hand, our approach, when applied to the generalized problem, may not always yield quantum speedup. An analysis shows that a large number of ground odd-states (respectively even-states) for perturbation $\mathcal{V}^{o}$ (respectively $\mathcal{V}^{e}$ ) makes the multiplicities of $b_{3}(t)$ and $b_{4}(t)$ in equation (9) differ to some extent. Thus the advantage of Algorithm 2 with respect to Algorithm 1 disappears. It would be interesting to know if our approach can be extended to handle this generalized problem with definite quantum speedup. Also whether our method has unseen advantages over other methods remains further investigation. 
Even though our perturbation Hamiltonian looks more complicated than that of ${ }^{(2)}$ it does not directly imply our proposal is harder to implement. The implementation issue is beyond the scope of our focus and poses a challenge to the experimentalists. Nevertheless, it at least suggests a new way of performing quantum search.

\section{CONCLUSIONS}

Motivated by developing a quantum search algorithm, we formulated a multi-state harmonic perturbation problem and gave an exact analysis. This paper answered affirmatively and constructively the open question whether fast time-varying Hamiltonians can be exploited in quantum search. The presented algorithm is optimal and achieves a quadratic speed-up over classical algorithms similar to prior methods. ${ }^{(1-3)}$ Under this new computation model, we hope it may suggest different approaches to the realization of quantum computers.

\section{APPENDIX}

Theorem 1

Proof. Expanding $\Lambda$ of equation (14), we have

$$
\begin{aligned}
\Lambda= & \frac{1}{\left(s+i \omega_{1}\right) \cdots\left(s+i \omega_{m}\right)}\left\{s^{m}+\cdots\right. \\
& \left.+\left(\sum_{j} \alpha_{j} \sum_{k \neq j}\left(\prod_{l \neq j, l \neq k} \omega_{l}\right)\right) s^{2}+\left(\sum_{j} \alpha_{j} \prod_{k \neq j} \omega_{k}\right) s\right\} .
\end{aligned}
$$

We first show that, if $d>0$, the largest exponent in terms of $\omega_{R}$ in the denominator of equation (15) must result from the product of all $\omega_{j}$ 's. Let $\sigma=x_{1}+\cdots+x_{m}$; then the product term $\omega_{1} \omega_{2} \cdots \omega_{m}$ equals $a \omega_{R}{ }^{\sigma}$ for some constant $a \neq 0$. For contradiction, suppose that the product term $\omega_{1} \omega_{2} \cdots \omega_{m}$ is not the only highest order term in $\omega_{R}$. Then there must exist at least one $\omega_{j}=a_{j} \omega_{R}{ }^{x_{j}}$ with $x_{j} \leq 0$. Let $a^{\prime} \omega_{R}^{\sigma^{\prime}}$ for $a^{\prime} \neq 0$ be the product term of all $\omega_{j}$ 's with $x_{j}>0$. Because $a^{\prime} \omega_{R}{ }^{\sigma^{\prime}}$ must appear somewhere in both the denominator and nominator of equation (15), $d$ must equal 0 . Hence, to have $d>0$, all $\omega_{j}$ 's must have $x_{j}>0$. That is, the product term $\omega_{1} \omega_{2} \cdots \omega_{m}$ contributes to the largest exponent of $\omega_{R}$ in the denominator.

To have $d>2$, the exponent of $\omega_{R}$ in the nominator of equation (15) cannot be too large. Observe that, for the resonance condition to 
hold in harmonic perturbation, there must exist some $x_{k}=1$. Moreover, there are more than one such $x_{k}$ 's. Otherwise, the product term $\omega_{1} \cdots \omega_{k-1} \omega_{k+1} \cdots \omega_{m}$ cannot be cancelled out. Its existence in the nominator of equation 15 results in $d \leq 1$, which violates the desired condition. Because of these constraints and $x_{j}>0$ for $j=1, \ldots, m$, the coefficients of $s$ and $s^{2}$ in the nominator of equation (15) must equal zero. That is,

$$
\begin{array}{r}
\sum_{j} \alpha_{j} \prod_{k \neq j} \omega_{k}=0, \text { and } \\
\sum_{j} \alpha_{j} \sum_{k \neq j}\left(\prod_{l \neq j, l \neq k} \omega_{l}\right)=0
\end{array}
$$

must be satisfied. Multiplying (16) by $\left(\frac{1}{\omega_{1}}+\cdots+\frac{1}{\omega_{m}}\right)$ yields

$$
\sum_{j} \alpha_{j} \frac{\prod_{k \neq j} \omega_{k}}{\omega_{j}}+\sum_{j} \alpha_{j} \sum_{k \neq j}\left(\prod_{l \neq j, l \neq k} \omega_{l}\right)=0 .
$$

By recognizing that the second term of equation (18) equals 0 by equation (17), it is immediate that

$$
\sum_{j} \alpha_{j} \frac{\prod_{k \neq j} \omega_{k}}{\omega_{j}}=0 .
$$

Again, multiplying equation (19) by the product term $\omega_{1} \omega_{2} \cdots \omega_{m}$ yields

$$
\sum_{j} \alpha_{j} \prod_{k \neq j} \omega_{k}^{2}=0 .
$$

Since $\alpha_{j}$ 's are positive real numbers, the left-hand side of equation (20) must be greater than zero unless all $\omega_{j}$ 's equal zero, which violates the condition that $\omega_{j} \neq \omega_{k}$ for $j \neq k$. Thus, equation (20) does not hold, and $d \ngtr 2$. Moreover, since $d=2$ is achieved by Algorithm 2, the theorem follows.

\section{REFERENCES}

1. L. Grover, in Proceedings of the 28th ACM Symposium on the Theory of Computing, pp. 212-219 (1996).

2. E. Farhi and S. Gutmann, Phys. Rev. A 57(4), 2403-2406 (1998).

3. E. Farhi, J. Goldstone, S. Gutmann, and M. Sipser, Quantum computation by adiabatic evolution, quant-ph/0001106 (2000). 
4. W. van Dam, M. Mosca, and U. Vazirani, in Proceedings of the 42th IEEE Symposium on Foundations of Computer Science, pp. 279-287 (2001).

5. J. Roland and N. Cerf, Phys. Rev. A 65(4), 042308(6) (2002).

6. D. Aharonov, W. van Dam, J. Kempe, Z. Landau, S. Lloyd, and O. Regev, in Proceedings of the 45th IEEE Symposium on Foundations of Computer Science (2004).

7. L. Vandersypen, M. Steffen, M. Sherwood, C. Yannoni, G. Breyta, and I. Chuang, Appl. Phys. Lett. 76(5), 646-648 (2000).

8. X. Peng, X. Zhu, X. Fang, M. Feng, M. Liu, and K. Gao, Phys. Rev. A 65, 042315 (2002).

9. M. Steffen, W. van Dam, T. Hogg, G. Breyta, and I. Chuang, Phys. Rev. Lett. 90(6), 067903(4) (2003).

10. J. Preskill, Proc. R. Soc. Lond. A 454, 469-486 (1998).

11. J. J. Sakurai, Modern Quantum Mechanics (Addison Wesley, 1994).

12. C. Bennett, E. Bernstein, G. Brassard, and U. Vazirani, Siam J. Comput. 26, 1277-1339 (1997).

13. A. Romanelli, A. Auyuanet, and R. Donangelo, Physica A 360, 274-284 (2006).

14. M. Boyer, G. Brassard, P. Hoeyer, and A. Tapp, Fortschritte der Physik 46, 493-506 (1998). 